\title{
A Safe and Effective Two-Step Tract Dilation Technique in Totally Ultrasound-Guided Percutaneous Nephrolithotomy
}

\section{Shao-Wei Dong}

Department of Urology, Shuang Ho Hospital, Taipei Medical University, New Taipei City

\section{Chia-Chang Wu}

Department of Urology, Shuang Ho Hospital, Taipei Medical University, New Taipei City

Chu-Tung Lin

Division of Urology, Department of Surgery, Shin Kong Wu Ho-Su Memorial Hospital, Taipei

\section{Kuan-Chou Chen}

Department of Urology, Shuang Ho Hospital, Taipei Medical University, New Taipei City

Chen-Hsun Ho ( $\nabla$ chho.uro@gmail.com )

Division of Urology, Department of Surgery, Shin Kong Wu Ho-Su Memorial Hospital, Taipei

\section{Research Article}

Keywords: Percutaneous Nephrolithotomy, Ultrasound, Urolithiasis, Fascial Dilator, Balloon Dilator

Posted Date: September 22nd, 2021

DOI: https://doi.org/10.21203/rs.3.rs-899484/v1

License: (a) This work is licensed under a Creative Commons Attribution 4.0 International License. Read Full License 


\section{A Safe and Effective Two-Step Tract Dilation Technique in Totally}

\section{Ultrasound-Guided Percutaneous Nephrolithotomy}

Shao-Wei Dong, ${ }^{1}$ Chia-Chang Wu, ${ }^{1,2}$ Chu-Tung Lin, ${ }^{3}$ Kuan-Chou Chen, ${ }^{1,2,4}$

Chen-Hsun $\mathrm{Ho}^{3,5}$

1. Department of Urology, Shuang Ho Hospital, Taipei Medical University, New Taipei City, Taiwan

2. Department of Urology, School of Medicine, College of Medicine, Taipei Medical University, Taipei, Taiwan

3. Division of Urology, Department of Surgery, Shin Kong Wu Ho-Su Memorial Hospital, Taipei, Taiwan

4. Graduate Institute of Clinical Medicine, College of Medicine, Taipei Medical University, Taipei

5. School of Medicine, College of Medicine, Fu Jen Catholic University, New Taipei City, Taiwan

Correspondence to: Chen-Hsun Ho, MD, PhD

Division of Urology, Department of Surgery, Shin Kong Wu Ho-Su Memorial Hospital, Taipei, Taiwan

Email: chho.uro@gmail.com

Phone: +886 228332211 ext 2082

Address: No. 95, Wen Chang Road, Shih Lin District, Taipei, Taiwan 


\section{Abstract \\ Background}

To evaluate the safety and the efficacy of a radiation-free 2-step tract dilation technique in totally ultrasound-guided percutaneous nephrolithotomy (PCNL).

\section{Methods}

From Oct 2018 to Mar 2020, we prospectively and consecutively enrolled 18 patients with 19 kidney units with urolithiasis. The nephrostomy tract was established by the following four steps: 1) ultrasound-guided renal puncture, 2) first-stage serial dilation to 16 Fr with Amplatz dilators, 3) check and adjustment of the partially dilated tract with a ureteroscope, 4) second-stage dilation with a 24-Fr balloon dilator.

\section{Results}

The median age was 62.0 [IQR 11.0] years, and $11(61.1 \%)$ were male. The median stone size was $3.3[3.6] \mathrm{cm}^{2}$, and stone laterality was almost equal over both sides. Successful tract establishment on the first attempt without fluoroscopy was achieved in $18(94.7 \%)$ operations. The median tract establishment time was 10.4 [4.9] mins, and the median operation time was 67.0 [52.2] mins. The median hemoglobin drop was $1.0[1.1] \mathrm{g} / \mathrm{dL}$, and none required blood transfusion. Three (15.8\%) developed fever. Pleural injury occurred in two (10.5\%) operations (both had supracostal puncture), and one required drainage with pigtail. Stone-free status was achieved in 15 (77.8\%) operations at 3 months postoperatively. 


\section{Conclusions}

Herein we present a radiation-free 2 -step tract dilation technique, which is characterized by ureteroscopic check of the partially dilated tract in between the first dilation with serial fascial dilators and the second dilation with balloon. Our data suggest that it is a safe and effective method.

\section{Keywords}

Percutaneous Nephrolithotomy · Ultrasound · Urolithiasis · Fascial Dilator · Balloon Dilator 


\section{Background}

Percutaneous nephrolithotomy (PCNL) has remained the treatment of choice for large and complex kidney stones, since it was first introduced in 1970 s(1). The step of establishing an access to the collecting system, including renal puncture and the subsequent tract dilation, is the crucial part of this surgery and significantly affects the outcome. Traditionally, fluoroscopy has been used to establish the nephrostomy tract in PCNL and to date it remains the most common image modality to guide $\mathrm{PCNL}(2)$. It facilitates the tract establishment by providing a clear mapping of the whole collecting system. Besides, almost all kinds of tract dilators, such as Amplatz, balloon, and Alken telescopic metal dilators, are designed to be visible under fluoroscopy, which ensures a successful access to the collecting system. Despite these advantages, there has been a persistent concern about the patients' and the operators' exposure to the ionizing radiation with fluoroscopic guidance(3).

Ultrasound-guided PCNL has gained a growing popularity in the past few years. It has the merit of reducing or even totally avoiding radiation exposure. Other advantages include visualization of the surrounding organs, prevention of vascular injury by Doppler imaging, no need of wearing lead apron, and so on(4). However, while it is usually not difficult to perform renal puncture under ultrasound, the subsequent tract dilation solely by ultrasound is considered challenging and sometimes impossible(5). This is because that almost all kinds of dilators are less echogenic and barely visible under ultrasound(6). In the literature, many so-called "ultrasound-guided" PCNLs actually refer to ultrasound-guided renal puncture followed by fluoroscopy-guided tract 
dilation(7-9). While some authors solely use ultrasound to monitor the advancement of Amplatz, telescopic metal, or balloon dilators $(10,11)$, these generally require an advanced ultrasound technique and are considered limited to experienced hands(12).

To overcome the above challenging tract dilation during totally ultrasound-guided PCNL, we utilized a radiation-free nephrostomy tract establishment technique in the past few years. The dilation was carried out in a 2-step manner, and in between the tract was checked by a quick look with a ureteroscope. As it did not require an advanced technique to monitor the dilators' advancement under ultrasound, this method should be much easier for surgeons with any level of ultrasound expertise. The objective of the current study was to evaluate the safety and the efficacy of this radiation-free 2-step tract dilation technique in totally ultrasound-guided PCNL.

\section{Methods}

Patient selection

From Oct 2018 to Mar 2020, we consecutively enrolled 18 subjects with 19 renal units with urolithiasis. A total 19 operations were included in the study. All the operations were performed by a single surgeon $(\mathrm{CHH})$. The study was approved by Taipei Medical University-Joint Institutional Review Board (N201808062).

Surgical techniques

Under endotracheal general anesthesia, the patient was first placed in a lithotomy position. A 5-Fr ureteral catheter (PAHSCO, Taiwan) was indwelled, 
which allowed infusion of normal saline to obtain an artificial hydronephrosis. The patient was then repositioned in a prone position. Ultrasonography (BK Medical ApS, Mileparken, Herlev, Denmark) was performed to identify the anatomy of the collecting system and stone position. The route of puncture was determined by the surgeon, and upper calyx was preferentially chosen in this series. Under ultrasound guidance, the targeted calyx was punctured with a 18-Ga/20-cm Chiba needle (Argon Medical Devices, Athens, TX, USA). A 0.035-inch J-tip guidewire (Boston Scientific, Marlborough, MA, USA) was then inserted into the collecting system through the Chiba needle. The depth of renal puncture was measured with the scale on the Chiba needle. On the first step of dilation, the tract was dilated serially with the 8-Fr, 10-Fr, 12-Fr, 14-Fr, and 16-Fr Amplatz Dilators (Boston Scientific, Spencer, IN, USA). All the Amplazt dilators was advanced at the previously measured depth. A 16- $\mathrm{Fr}$ Amplatz Sheath (Boston Scientific, Spencer, IN, USA) was then passed over the 16-Fr dilator into the collecting system.

A semi-rigid 6.0/7.5-Fr ureteroscope (Richard Wolf, Vemon Hills, Illinois, USA) was advanced through the Amplatz sheath to check whether the tract appropriately entered the collecting system. If necessary, the sheath was advanced further along the shaft of the ureteroscope to completely penetrate the renal parenchyma. This ensured the success of the second step of dilation, in which a 24-Fr nephrostomy balloon dilator (Ultraxx ${ }^{\mathrm{TM}}$, Cook, Bloomington, IN, USA) was inserted through 16-Fr Amplatz sheath into the collecting system. With the balloon dilator in place, the Amplatz sheath was withdrawn and the balloon was inflated with a pressure of $14 \mathrm{~atm}$ for $30 \mathrm{sec}$. A 24-Fr sheath was then advanced over the inflated balloon. Once the tract was 
established, the stones were fragmented with pneumatic lithotripter or holmium laser, and the stone fragments were retrieved with a grasper. In the end of the surgery, a 6-Fr double-J ureteral stent was indwelled and a 20-Fr nephrostomy tube was placed to conclude the operation.

Data collection

Study subjects' demographics and data regarding the stone, including size, location, and composition, were recorded. Successful tract establishment was defined as the distal end of the 24-Fr working sheath appropriately placed in the collecting system solely under ultrasound on the first attempt. Cases in which second dilation procedure was required or fluoroscopy was applied at any time of tract establishment were considered as failures. Tract establishment time was calculated from the beginning of renal puncture to the successful placement of the 24-Fr working sheath. Operative time was measured from the retrograde ureteral catheterization to the nephrostomy tube insertion. Any postoperative events and complications were recorded. Stone-free status was defined as no residual fragments greater than $4 \mathrm{~mm}$ determined by KUB or CT at 3 months postoperatively.

Statistical analysis

The continuous variables are expressed as the median and [interquartile range (IQR)]; the categorical variables are expressed as count and (percentage). The statistics was carried out with an intention-to-treat analysis. The analysis was conducted with SPSS 26.0 for Windows (SPSS Inc., USA). 


\section{Results}

The characteristics of the study subjects are shown on Table 1. The median age was 62.0 [11.0] years, and $11(61.1 \%)$ were male. The median stone size was $3.3[3.6] \mathrm{cm}^{2}$, and stone was located on the right side in 9 $(47.4 \%)$ subjects. Renal access was made on the upper calyx in $17(89.5 \%)$ operations.

Successful tract establishment on the first attempt was achieved in 18 $(94.7 \%)$ operations. In one case (5.3\%), unintentional withdraw of the guidewire occurred on the first stage of dilation (serial Amplatz), in which we failed to regain an artificial hydronephrosis and fluoroscopy was applied to complete tract establishment. The median tract establishment time was 10.4 [4.9] mins. The median operation time was 67.0 [52.2] mins. The overall and postoperative hospital stays were 3.9 [4.0] days and 2.7 [3.9] days, respectively. The median hemoglobin drop was $1.0[1.1] \mathrm{g} / \mathrm{dL}$, and none required blood transfusion (Table 2).

Postoperatively, pyuria was noted in eight (42.1\%) operations: the majority was asymptomatic and only three (15.8\%) developed fever. Pleural injury occurred in two (10.5\%) operations, in both supracostal puncture was made to gain access to the upper calyx. One was asymptomatic and was conservatively treated, while the other was symptomatic and was successfully treated with pigtail drainage. Stone-free status was achieved in 15 (78.9\%) operations at 3 months after operation. Three (15.8\%) underwent an ancillary procedure of extracorporeal shockwave lithotripsy or ureteroscopy.

\section{Discussion}


In the current study, we described a technique of radiation-free tract establishment in totally ultrasound-guided PCNL. The collecting system was punctured under real-time ultrasound guidance. Then, the tract was dilated in a 2-step technique. The tract was initially dilated from $8 \mathrm{Fr}$ to $16 \mathrm{Fr}$ with Amplatz fascial dilators. Then, the initially dilated tract was checked and adjusted with a ureteroscope. Finally, the tract was further dilated with a 24-Fr balloon dilator. The results demonstrated that the above technique achieved a high success rate of tract establishment on the first attempt, without fluoroscopy. And the time required to establish the tract was consistently short. Compared to ultrasound-guided tract dilation, this method did not require an advanced ultrasound technique and was considered to be easier to carry out.

Several mechanisms contributed to the safety and the efficacy of this technique. First, as the tips of small-sized Amplatz dilators (8 Fr to $16 \mathrm{Fr}$ ) are relatively sharp, which tend to successfully penetrate the kidney parenchyma and enter the collecting system. This largely prevented the status of short dilation. This was supported by those studies of mini-PCNL $(13,14)$, in which the failure rate of serial dilation up to $18 \mathrm{Fr}$ was generally less than $5 \%$. On the contrary, the tips of large-sized Amplatz dilators are relatively blunt and tend to push the kidney away before they enter the collecting system, which causes a status of short dilation $(15,16)$. Second, all the small-sized (8-Fr to 16-Fr) Amplatz dilators were advanced at a fixed depth as measured earlier. This avoided the injury to the opposite pelvic membrane in the collecting system. With the above two mechanisms, we could confidently dilate the tract until a diameter of $16 \mathrm{Fr}$ was reached without monitoring by either fluoroscopy 
or ultrasound. Third, after the tract was initially dilated, we checked the entrance of the 16-Fr tract with a ureteroscope. In our experience, the 16-Fr sheath had been perfectly placed in the collecting system at this step in the majority. Although short dilation did occur in a few cases, it was not difficult to fix by the following procedure: following the guidewire, the ureteroscope was advanced into the collecting system; then the $16-\mathrm{Fr}$ sheath was gently advanced over the shaft of the ureteroscope. The appropriately positioned 16Fr sheath almost ensured the success of the final dilation with a balloon.

A few authors reported their technique of ultrasound monitoring the dilation process, including balloon dilator(11, 17), serial Amplatz dilators(9), one-shot Amplatz dilators(18), or telescopic metal (Alken) dilators(19). Among these dilators, balloon has been widely used in ultrasound-guided PCNL and has been considered relatively easier to apply under ultrasound guidance(12). In one study with 138 subjects, totally ultrasound-guided balloon dilation was achieved in 131 (94.9\%), and the remaining 7 (5.1\%) cases required a switch to fluoroscopy(17). In another study with 207 cases undergoing ultrasoundguided balloon dilation, the success rate of tract dilation on the first attempt was $88.4 \%(11)$. Short dilation was considered to be the main cause of failure with balloon dilation $(15,16)$. Obesity was reported to predict failure of ultrasound-guided balloon dilation, as it was reported that the success rate was $76.9 \%$ in normal weight and $79.0 \%$ in overweight, but it was only $45.7 \%$ in obese subjects(6). In another study, the presence of staghorn stones, previous ipsilateral open nephrolithotomy, and low pole access independently predicted a failure of tract establishment with balloon, while the presence of hydronephrosis of the target calyx increased the likelihood of success(11). In 
general, to monitor these dilators under ultrasound is an advanced technique and is limited to experts and is specially technically challenging in cases without hydronephrosis(16).

Different from the above techniques by using ultrasound to monitor the process of dilation, our method was characterized by checking the initial small-sized dilation with a ureteroscope. This was considered as a 2-step technique in the literature. Wang et al.(11) reported their 2-step dilation technique. The tract was first serially dilated from 8-Fr to $16-\mathrm{Fr}$ with fascial dilators. A 16-Fr peel-away sheath was left to allow the evaluation by ureteroscope. In the second step, a 15-Fr metal dilator was inserted to replace the peel-away sheath, followed by further dilation with 18 to 24-Fr metal dilator. Compared to one-step balloon dilator, their 2-step technique achieved a significantly higher success rate of tract establishment on the first attempt (100\% vs $88.6 \%)$, and the mean tract establishment time was only 6 minutes(11). The same technique was also reported by Song et al.(20), who reported a success rate of $84.3 \%$ on the first attempt. In another technique of Zhou et al.(5), a 10-Fr fascial dilator was first used to dilate the tract along the guidewire, and a 10-Fr sheath was left in place. A 6-Fr ureteroscope entered the collecting system to adjust the guidewire into the ureter. Then the tract was further dilated with either a balloon dilator or serial fascial dilator (from 12 Fr to $22 \mathrm{Fr}$ ). With either type of dilator, the success rate of tract establishment was high and the mean tract establishment time was short (8.9 mins versus 10.1 mins). The success rate and the tract establishment time in our study were comparable to the those of the above studies $(5,11,20)$. Our study generally confirmed that the safety and the efficacy of the 2-step technique 
were at least not inferior to those of fluoroscopy-guided or ultrasound-guided tract dilation, while it maintained the merits of no radiation and lower ultrasound expertise required.

The major limitation of the current study was a small case number. However, we believed that the results were still robust enough to support the feasibility of this technique. First, among the 19 consecutive operations, there was only one failure, which was due to an unintentional withdraw of the guidewire. This should not be considered as a failure of the technique itself. Second, the time of tract establishment was consistent and short, suggesting that this technique was easy to perform and was highly reproducible. Third, the overall complication rates, regarding the blood loss, transfusion rate, and fever were generally low and were comparable to those in the literature. There was a relatively higher rate of pleural injury, which was because that upper calyx was the primary target of puncture (supracostal) in the majority of the cases.

\section{Conclusions}

Herein we present a radiation-free tract dilation method. It is a 2-step technique: in between the first dilation with serial fascial dilators and the second dilation with balloon, the partially dilated tract is checked and adjusted with ureteroscope. Our data suggest that it is a safe and effective method.

\section{List of abbreviations}

PCNL: Percutaneous nephrolithotomy 


\section{Declarations}

\section{Ethics approval and consent to participate}

The study was approved by Taipei Medical University-Joint Institutional Review Board (N201808062). All the patients agreed to participate in the study and signed the informed consent. All methods were carried out in accordance with relevant guidelines and regulations.

\section{Consent for publication}

Not applicable

\section{Availability of data and materials}

The datasets generated and/or analysed during the current study are not publicly available due to limitations of ethical approval involving the patient data and anonymity but are available from the corresponding author on reasonable request.

\section{Competing interests}

The authors declare that they have no competing interests

\section{Funding}

This work was supported by the grant from Shin Kong Wu Ho-Su Memorial Hospital (2021SKHBDR001)

\section{Authors' contributions}

Study Concept and Design: SWD, $\mathrm{CHH}$; Acquisition of Data: SWD, $\mathrm{CHH}$; 
Analysis data: SWD; Drafting of the manuscript: SWD; Substantively revised the work: CCW, CTL, KCC, $\mathrm{CHH}$. All authors have read and approved the manuscript.

\section{Acknowledgments}

Not applicable.

\section{References}

1. Fernstrom I, Johansson B. Percutaneous pyelolithotomy. A new extraction technique. Scand J Urol Nephrol. 1976;10(3):257-9.

2. Andonian S, Scoffone CM, Louie MK, Gross AJ, Grabe M, Daels FP, et al. Does imaging modality used for percutaneous renal access make a difference? A matched case analysis. J Endourol. 2013;27(1):24-8.

3. Corrales M, Doizi S, Barghouthy Y, Kamkoum H, Somani B, Traxer O.

Ultrasound or Fluoroscopy for Percutaneous Nephrolithotomy Access, Is There Really a Difference? A Review of Literature. J Endourol. 2021;35(3):241-8.

4. Lojanapiwat B. The ideal puncture approach for PCNL: Fluoroscopy, ultrasound or endoscopy? Indian J Urol. 2013;29(3):208-13.

5. Zhou T, Chen G, Gao X, Zhang W, Xu C, Li L, et al. 'X-ray'-free balloon dilation for totally ultrasound-guided percutaneous nephrolithotomy. Urolithiasis. 2015;43(2):189-95.

6. Usawachintachit M, Masic S, Chang HC, Allen IE, Chi T. Ultrasound Guidance to Assist Percutaneous Nephrolithotomy Reduces Radiation Exposure in Obese Patients. Urology. 2016;98:32-8.

7. Ng FC, Yam WL, Lim TYB, Teo JK, Ng KK, Lim SK. Ultrasound-guided 
percutaneous nephrolithotomy: Advantages and limitations. Investig Clin Urol. 2017;58(5):346-52.

8. Basiri A, Ziaee AM, Kianian HR, Mehrabi S, Karami H, Moghaddam SM. Ultrasonographic versus fluoroscopic access for percutaneous nephrolithotomy: a randomized clinical trial. J Endourol. 2008;22(2):281-4.

9. Ding $X$, Hao $Y$, Jia $Y$, Hou $Y$, Wang C, Wang Y. 3-dimensional ultrasound-guided percutaneous nephrolithotomy: total free versus partial fluoroscopy. World J Urol. 2020;38(9):2295-300.

10. Tzou DT, Metzler IS, Usawachintachit M, Stoller ML, Chi T. Ultrasound-guided Access and Dilation for Percutaneous Nephrolithotomy in the Supine Position: A Step-by-Step Approach. Urology. 2019;133:245-6.

11. Wang $\mathrm{S}$, Zhang $\mathrm{Y}$, Zhang $\mathrm{X}$, Tang $\mathrm{Y}, \mathrm{Xiao} \mathrm{B}, \mathrm{Hu} \mathrm{W}$, et al. Tract dilation monitored by ultrasound in percutaneous nephrolithotomy: feasible and safe. World J Urol. 2020;38(6):1569-76.

12. Beiko D, Razvi H, Bhojani N, Bjazevic J, Bayne DB, Tzou DT, et al. Techniques Ultrasound-guided percutaneous nephrolithotomy: How we do it. Can Urol Assoc J. 2020;14(3):E104-E10.

13. Xiong L, Huang X, Ye X, Chen L, Ma K, Liu J, et al. Microultrasonic Probe Combined with Ultrasound-Guided Minipercutaneous Nephrolithotomy in the Treatment of Upper Ureteral and Renal Stones: A Consecutive Cohort Study. J Endourol. 2020;34(4):429-33.

14. Zhu W, Li J, Yuan J, Liu Y, Wan SP, Liu G, et al. A prospective and randomised trial comparing fluoroscopic, total ultrasonographic, and combined guidance for renal access in mini-percutaneous nephrolithotomy. BJU Int. 2017;119(4):612-8. 
15. Pakmanesh H, Daneshpajooh A, Mirzaei $M$, Shahesmaeili A, Hashemian $M$, Alinejad $\mathrm{M}$, et al. Amplatz versus Balloon for Tract Dilation in Ultrasonographically Guided Percutaneous Nephrolithotomy: A Randomized Clinical Trial. Biomed Res Int. 2019;2019:3428123.

16. Jin $\mathrm{W}$, Song $\mathrm{Y}$, Fei $\mathrm{X}$. The Pros and cons of balloon dilation in totally ultrasound-guided percutaneous Nephrolithotomy. BMC Urol. 2020;20(1):82.

17. Armas-Phan M, Tzou DT, Bayne DB, Wiener SV, Stoller ML, Chi T. Ultrasound guidance can be used safely for renal tract dilatation during percutaneous nephrolithotomy. BJU Int. 2020;125(2):284-91.

18. Karami H, Rezaei A, Mohammadhosseini M, Javanmard B, Mazloomfard M, Lotfi B. Ultrasonography-guided percutaneous nephrolithotomy in the flank position versus fluoroscopy-guided percutaneous nephrolithotomy in the prone position: a comparative study. J Endourol. 2010;24(8):1357-61.

19. Hosseini MM, Hassanpour A, Farzan R, Yousefi A, Afrasiabi MA. Ultrasonography-guided percutaneous nephrolithotomy. J Endourol. 2009;23(4):6037.

20. Yan S, Xiang F, Yongsheng S. Percutaneous nephrolithotomy guided solely by ultrasonography: a 5-year study of >700 cases. BJU Int. 2013;112(7):965-71. 


\section{Table Legends}

Table.1 Characteristics of the Study Subjects

Table 2. Outcomes of Totally Ultrasound-Guided PCNL

Supplement Video. Demonstration of the two-step radiation-free technique of tract dilation in totally ultrasound-guided PCNL 


\section{Supplementary Files}

This is a list of supplementary files associated with this preprint. Click to download.

- Table2.docx.pdf

- Table1.docx.pdf

- pcnl03.mp4 